\title{
LDL-C Oxidation
}

National Cancer Institute

\section{Source}

National Cancer Institute. LDL-C Oxidation. NCI Thesaurus. Code C129899.

The presence of oxidized elements in cholesterol-bearing low-density lipoprotein (LDL) particles in the blood. Oxidation may be associated with increased risk for atherosclerosis. 